\title{
Emmanuel Dias: o principal artífice do combate à doença de Chagas nas Américas
}

\author{
Emmanuel Dias: the principal architect of the fight \\ against Chagas disease in the Americas
}

\author{
João Carlos Pinto Dias ${ }^{1,2}$, Aluízio Prata ${ }^{2,3,4}$ e José Rodrigues Coura ${ }^{4,5}$
}

\begin{abstract}
RESUMO
Comemora-se em 2008 o centenário de nascimento do Dr. Emmanuel Dias, cuja vida foi intensamente dedicada ao estudo, reconhecimento e controle da doença de Chagas. Esta súmula se incorpora às diversas homenagens feitas no Brasil e no Exterior, resgatando alguns elementos históricos na trajetória do cientista e o enorme impacto social resultante direta e indiretamente de seu trabalho. Afilhado e assistente de Carlos Chagas, Emmanuel foi considerado por Chagas Filho como o mais profícuo dos seguidores de seu pai. Em trinta anos de atividades, Dias iniciou-se como brilhante protozoologista depois passando ao enfrentamento da doença humana. Trabalhou intensivamente em diagnóstico, epidemiologia, clinica e controle. Idealizou estratégias de prospecção, mapeou a doença nas Américas, participou diretamente da sistematização da cardiopatia crônica e descreveu o primeiro inseticida eficaz contra os triatomíneos, também formatando a estratégia de seu controle. Mais ainda, estendeu suas atividades para toda a área endêmica, divulgando a doença e seu controle, de um lado, e impulsionando autoridades sanitárias e centros de decisão com vistas a implantação de ações, de outro. De seu trabalho resultaram programas nacionais e regionais que reduziram significativamente a transmissão da doença humana em todo o Continente. Foi recentemente considerado como o cientista que teve o maior impacto no enfrentamento desta tripanossomíase, em todos os tempos.
\end{abstract}

Palavras-chaves: Emmanuel Dias. Doença de Chagas. Controle.

\begin{abstract}
In 2008, the centenary of the birth of Emmanuel Dias, whose life was intensely dedicated to the study, recognition and control of Chagas disease, is being celebrated. This summary of his life joins with the various homages paid in Brazil and abroad, to recall some of the key historical points in this scientist's career and the enormous social impact that resulted directly and indirectly from his work. Dias, who was Carlos Chagas' protégée and assistant, was considered by Chagas Filho to be the most proficient of his father's followers. Over the course of thirty years of activities, Dias began as a brilliant protozoologist and later on turned his attention towards facing the human disease. He worked intensively on diagnostics, epidemiology, clinical studies and control. He devised prospection strategies, mapped out the disease in the Americas, participated directly in systematizing chronic cardiopathy and described the first effective insecticide against triatomines, along with laying out the strategy for their control. Furthermore, he extended his activities throughout the field of this endemic disease: on the one hand, raising awareness about the disease and its control and, on the other hand, propelling health authorities and decision-making centers into action, to implement control measures. His work resulted in national and regional programs that have significantly reduced the transmission of the human disease throughout the continent. Dias was recently said to be the scientist who had had the all-time greatest impact on this trypanosomiasis.
\end{abstract}

Key-words: Emmanuel Dias. Chagas disease. Control.

Em 2008, comemora-se o centenário de nascimento deste importante seguidor de Carlos Chagas. Filho do Dr. Ezequiel Caetano Dias, Emmanuel estudou na Faculdade Nacional de Medicina (Rio de Janeiro), defendendo tese doutoral sobre o Schizotrypanum cruzi em 1933, por ela merecendo os prêmios Gunning e de Louvor. Discípulo dileto e afilhado de Carlos Chagas, fez Curso de Aplicação e concurso público no Instituto Oswaldo Cruz, praticamente herdando o laboratório do padrinho, onde se dedicou brilhantemente aos estudos sobre o parasito e os vetores da tripanossomíase americana. Em 1935, foi com Evandro Chagas ao Congresso da MEPRA em Mendoza, Argentina, onde se inteirou da descrição de Romaña sobre o complexo oftalmo ganglionar, passando a divulgá-lo. Este sinal possibilitou a descoberta do importante foco de doença de Chagas em Bambuí, Minas Gerais, pelo Dr. Amílcar Vianna Martins, em outubro de 1940. Dias visitou imediatamente o foco e prognosticou que dali viriam

1. Centro de Pesquisa René Rachou, Fundação Oswaldo Cruz, Belo Horizonte, MG. 2. Academia Mineira de Medicina, Belo Horizonte, MG. 3. Disciplina de Doenças Infecciosas e Parasitárias, Faculdade de Medicina, Universidade Federal do Triângulo Mineiro, Uberaba, MG. 4. Academia Nacional de Medicina, Rio de Janeiro, RJ. 5. Departamento de Medicina Tropical, Instituto Oswaldo Cruz, Fundação Oswaldo Cruz, Rio de Janeiro, RJ.

Address to: Dr. João Carlos Pintos Dias. Centro de Pesquisa René Rachou/FIOCRUZ. Av. Augusto de Lima 1715, 30190-000 Belo Horizonte, MG.

Tel: 5531 3399-7763

e-mail: jcpdias@cpqrr.fiocruz.br

Recebido para pubicação em 10/09/2008

Aceito em 19/09/2008 
importantíssimos conhecimentos sobre a doença e seu controle. Conseguiu, com Evandro, ajuda no Instituto Oswaldo Cruz, para que o Instituto Ezequiel Dias levasse à frente um amplo projeto de pesquisa no Oeste de Minas. Artes do destino, Amílcar vai para a guerra na Itália e Emmanuel é convocado por seu Diretor, Dr. Henrique Aragão, para criar um Centro de Estudos $e$ Profilaxia da Doença de Chagas, em Bambuí, inaugurado em dezembro de $1943^{2478911}$

Neste trajeto, o brilhante parasitologista se depara com uma realidade humana e nosológica altamente impactante e totalmente negligenciada, mesclada de ranchos paupérrimos, elevadas taxas de infestação por Triatoma infestans e Panstrongylus megistus, abundantes casos agudos, cardiopatias severíssimas e morte súbita. Já em 1945, apoiado por Aragão e pelo Ministro Gustavo Capanema, desdobrava-se com seus companheiros Laranja, Nóbrega, Pellegrino e Dilermando Alves em duas frentes principais: de um lado mapeava e combatia os barbeiros, experimentando melhoramentos habitacionais e as mais diferentes armas químicas e físicas (soda cáustica, piretro, querosene, rotenona, gás cianídrico e lança chamas $)^{48911}$. De outro, desbravava-se a enfermidade, descrevendo-a de forma precisa e sistematizando o seu diagnóstico, com isso a colocando na compreensão de todos os clínicos da área endêmica ${ }^{61011}$. Houve grandes esperanças com o dicloro-difenil-tricloretano (DDT), que revolucionara a luta antimalárica em 1945, mas que falhou contra os triatomíneos. Em 1947, Dias e Pellegrino provam o gammexane (BHC), de extrema eficiência e com efeito residual próximo aos noventa dias, logo prognosticando o controle definitivo dos transmissores da esquizotripanose $\mathrm{e}^{151213}$.

Emmanuel Dias ficou em Bambuí até 1962, quando faleceu em pleno trabalho, de um acidente automobilístico. Do excelente pesquisador básico, as circunstâncias fizeram emergir um homem de serviço, com extrema determinação e incrível capacidade de trabalho, cada vez mais dedicado a um desideratum que lhe viera do próprio Dr. Chagas: "É preciso acabar com esta doença!". Para tanto, não se limitou a descrever a situação epidemiológica e a definir os meios de combate ao transmissor. Foi buscar as bases epidemiológicas e clínico-sociais que lhe possibilitassem, como aconselhado pelo amigo Fred Soper, da Pan American Health Organization (PAHO), a vender a idéia do controle aos governos e centros de decisã $0^{79}$. Amealhou parceiros preciosos por toda a área endêmica, o que lhe possibilitou mapear a doença pela América Latina, em trabalho meticuloso da década de 1950. Com enorme credibilidade, desenvolveu a estratégia de luta antivetorial e a propagou por meio de incansáveis conferências e consultorias pelo Continente, alternando Congressos com Gabinetes de autoridades, infindáveis trabalhos de campo, observações clínicas e experimentos em laboratório.

Publicou cerca de cento e oitenta trabalhos em praticamente trinta anos de atividade, média esta considerada excelente até para os nossos dias². Objetivava um controle integral da transmissão vetorial da doença de Chagas, mas também esteve atento aos bancos de sangue, com advertências pioneiras neste sentido, ainda nos anos quarenta ${ }^{8}$. Com três grandes aliados impulsionou de vez o controle da doença nas Américas: a) Mário Pinotti: Diretor do Departamento Nacional de Endemias Rurais (DNERu) e posteriormente Ministro da Saúde do Brasil, que apoiou incondicionalmente o amigo Emmanuel, fosse com veículos, insumos e funcionários, fosse com seu prestígio pessoal ou com a abertura e ampliação de frentes de trabalho; b) Arnoldo Gabaldón: eminente malariologista venezuelano, também Ministro da Saúde em seu País. Praticamente, instaurou, assessorado por Dias, a luta antitriatomínica na Venezuela, valendo-se do poderoso Serviço de Malariologia e de um ambicioso programa de melhorias habitacionais nas áreas endêmicas; c) Amador Neghme: o mais distinguido dos parasitologistas chilenos, que se tornou, com seu dileto assistente Hugo Schenone, ardoroso apologista das idéias e estratégias de Emmanuel, por eles implantadas em várias regiões do País Andino. Dias também fez discípulos e parceiros na Bolívia (com Torrico), no Peru (com Herrer), no Uruguai (com Talice e Franca), na Costa Rica (com Zeledón), entre outros. Talvez tenha sido seu querido México o país em que mais investiu, ali trabalhando com Mazotti, Biagi e os cardiologistas Ignacio Chavez, Sodi Pallares e Cabrera. Lamentavelmente, a terra azteca foi a última nação latinoamericana a reconhecer e priorizar a esquizotripanose $^{2491112}$.

Quando faleceu, em pleno apogeu de idéias e entusiasmo, almejava ver o Triatoma infestans erradicado de nosso País, uma idéia que ele acalentou já na década de 1950, tão logo avaliou os resultados que obteve em Bambuí e no Triângulo Mineiro. O Brasil recebeu em junho de 2006 a certificação da eliminação da transmissão da doença pelo Triatoma infestans, da Organização Pan-americana de Saúde/Organização Mundial de Saúde (OPS/OMS), ali se cumprindo o vaticínio de Emmanuel Dias, aliás homenageado pelo Ministro da Saúde, na ocasião. Dias também observara um decréscimo da morbidade crônica nas áreas trabalhadas, o que foi depois atestado por Vanize Macedo e outros pesquisadores ${ }^{6}$. Ainda em seus sonhos estaria um trabalho integrado de luta contra a doença, envolvendo os países latinoamericanos e a OPS. Passaram-se trinta anos da sua morte para que tal integração de fato se concretizasse, fruto de gente que se empenhou como Dias para construir as chamadas Iniciativas. Gabriel Schmunis, Pilar Alderette, Christopher Schofield, Roberto Salvatella, Carlos Chagas Filho e vários outros são alguns desses nomes, todos eles espelhados na luta pioneira de Emmanuel ${ }^{789}$.

No plano pessoal, Emmanuel Dias teve que enfrentar muitos e continuados sacrifícios para levar a cabo sua luta. Nos últimos vinte anos de sua vida trocou literalmente sua ativa e promissora vida no Rio de Janeiro pela efervescente e interminável lida em toda a área endêmica, centrada em Bambuí, sem morada fixa e sem férias regulares. Para tanto, levou a esposa Nícia e os cinco filhos para Belo Horizonte, reconhecendo nela o papel fundamental do zelo pela família. Emmanuel amava a vida, a boa comida, os bons amigos, a música clássica. Sabia ser feliz onde estivesse, ficando à vontade em lindos hotéis, em gabinetes ministeriais, em reuniões científicas e em modestas vendas do interior, onde houvesse um frango com arroz. Nunca reclamou de cansaço ou de excesso de trabalho. Não perdia tempo, trabalhando ou estudando em casa, em aviões ou no trenzinho de Bambuí. Guardava, com incomensurável respeito e carinho, 
as memórias de seu pai, de Carlos Chagas, de Eurico Villela e de Henrique Aragão, suas maiores referências. Manteve inabalável fidelidade ao seu amigo Mário Pinotti, no seu desterro e tempos de esquecimento.

Fez muitos discípulos e seguidores por toda a América. Tinha o dom do convencimento e do entusiasmo, que levou a cientistas e governantes. Estava tão convencido da prioridade e da factibilidade do controle dos triatomíneos que a isto se dedicou com extremo afinco nos últimos anos de sua vida. A única concessão, dizia, seria dedicar um tempo para seguir a história natural da doença de Chagas entre os mais de trezentos casos agudos registrados em Bambuí, projeto esse que segue vigente, levado a cabo no Centro Emmanuel Dias ${ }^{6}$. Um outro desdobramento ao controle, já prognosticado por Dias, seria a etapa de consolidação do programa, ou seja, a vigilância, de caráter permanente, horizontalizado e participativo. Este projeto também se implantou em Bambuí, pioneiramente em 1974, envolvendo a população, o governo local e agentes municipalizados de saúde. Posteriormente, este esquema serviu de modelo para toda a área endêmica ${ }^{56713}$.

Ao ensejo das comemorações do centenário de Emmanuel Dias (que coincide com o da descoberta do Trypanosoma cruzi), muitas memórias afloraram entre aqueles que o conheceram ou dele aprenderam alguma coisa. No Instituto Oswaldo Cruz, ao inaugurar-se um belo anfiteatro com seu nome, em 15 de agosto de 2008, foi relembrada a trajetória de um homem que se determinou em seu destino e soube, no momento certo, ampliar sua vocação acadêmica para uma pesquisa-ação de extraordinária importância social ${ }^{2}$. Foi uma existência ligada, indiscutivelmente, à saúde e à vida de milhões de cidadãos latinoamericanos. Secundando Carlos Chagas Filho, que afirmava ter sido ele o mais profícuo dos seguidores de seu pai, o Presidente da Fundação Oswaldo Cruz, Dr. Paulo Marchiori Buss, proclamou Emmanuel Dias como o cidadão e cientista que mais impacto teve no enfrentamento da doença de Chagas, em todos os tempos (PM Buss: discurso na FIOCRUZ, Rio de Janeiro, 15/8/2008).

Pouco após sua morte (1962), foi consolidada em Ribeirão Preto a fundação da Sociedade Brasileira de Medicina Tropical. Naquela ocasião, Emmanuel Dias foi homenageado, com emoção e respeito, por todos os circunstantes. A jovem Sociedade, a partir de então, iria cumprir um dos mais fundamentais papéis acadêmicos e políticos quanto ao desenvolvimento e a execução das lutas contra as doenças infecciosas e parasitárias, nas Américas e alhures. A doença de Chagas, hoje em vias de avançado controle, tem merecido da Sociedade Brasileira de Medicina Tropical um destaque todo especial. Em tal contexto, rememorar Emmanuel Dias e sua trajetória, faz-se um ato justo, oportuno e necessário, especialmente como exemplo para as novas gerações de tropicalistas.

\section{REFERÊNCIAS}

1. Coura JR. A historical review of Chagas Disease. In: Gilles HM (org) Protozoal Diseases. Arnold Editora, London, p. 306-312, 1999.

2. Coura JR. Contribuição de Emmanuel Dias ao estudo do Trypanosoma cruzi e da doença de Chagas e seu controle. 2008.

3. Dias E. Estudos sobre o Schizotrypanum cruzi. Memórias do Instituto Oswaldo Cruz 8: 1-115, 1934.

4. Dias E. 0 Centro de Estudos e Profilaxia de Moléstia de Chagas, em Bambuí, Estado de Minas Gerais. Notícia histórica em homenagem ao professor Henrique Aragão. Memórias do Instituto Oswaldo Cruz 54: 309-357, 1956.

5. Dias E. Profilaxia da doença de Chagas. Hospital 51: 285-298: 1957.

6. Dias JCP. Doença de Chagas em Bambuí, Minas Gerais, Brasil. Estudo clínico epidemiológico a partir da fase aguda entre 1940 e 1982 . Tese de Doutorado. Universidade Federal de Minas Gerais, Belo Horizonte, 1982.

7. Dias JCP. 0 controle da doença de Chagas no Brasil. In: Silveira AC (ed) El control de la enfermedad de Chagas en los países del Cono Sur de América Historia de una iniciativa internacional. 1991/2001. Uberaba: Organização Panamericana de Saúde, Faculdade de Medicina do Triângulo Mineiro, p. 146-250, 2002.

8. Dias JCP, Schofield CJ. Control of Chagas Disease. In: Maudlin I, Holmes PH, Miles MA (orgs) The Trypanosomes. CABI Publishing, London, p. 547-564, 2004.

9. Kropf SP. Doença de Chagas, doença do Brasil: ciência, saúde e nação (1909-1962). Tese de Doutorado. Universidade Federal Fluminense, Niterói, 2006.

10. Laranja FS, Dias E, Nóbrega GC, Miranda A. Chagas' disease. A clinical, epidemiologic and pathologic study. Circulation 14: 1035-1060, 1956.

11. Prata AR. Emmanuel Dias e a doença de Chagas. 2008.

12. Salvatella RA. Una visión de La enfermedad de Chagas desde su propia historia. In: Organizaçcion Panamericana de la Salud, Fundación Mundo Sano (orgs) La enfermedad de Chagas a la puerta de $\operatorname{los} 100$ años del conocimiento de una endemia americana ancestral. OPS/CD/426-06, Washington, p. 19-22, 2006.

13. World Health Organization. Control of Chagas Disease. Second report of the WHO Expert Committee. WHO Technical Report Séries 905, Geneva, 2002. 